\title{
Linx
}

Revue des linguistes de l'université Paris X Nanterre

38 | 1998

L'acquisition de la temporalité en situation bilingue

\section{Le développement de la subordination temporelle chez un enfant bilingue français-allemand (étude de cas)}

The acquisition of temporal subordination by a French-German bilingual child

\section{Ricarda Schneider}

\section{OpenEdition}

\section{Journals}

Édition électronique

URL : http://journals.openedition.org/linx/894

DOI : 10.4000/linx.894

ISSN : 2118-9692

\section{Éditeur}

Presses universitaires de Paris Nanterre

\section{Édition imprimée}

Date de publication : 1 décembre 1998

Pagination : 171-193

ISSN : 0246-8743

\section{Référence électronique}

Ricarda Schneider, «Le développement de la subordination temporelle chez un enfant bilingue français-allemand (étude de cas) », Linx [En ligne], 38| 1998, mis en ligne le 02 juillet 2012, consulté le 21 avril 2019. URL : http://journals.openedition.org/linx/894 ; DOI : 10.4000/linx.894

Ce document a été généré automatiquement le 21 avril 2019.

Département de Sciences du langage, Université Paris Ouest 


\title{
Le développement de la subordination temporelle chez un enfant bilingue français-allemand (étude de cas)
}

The acquisition of temporal subordination by a French-German bilingual child

\author{
Ricarda Schneider
}

\section{Introduction}

1 Ce travail est une étude de cas qui se propose d'analyser le développement de la subordination temporelle par un enfant acquérant le français et l'allemand dans un cadre familial. Dès sa naissance, les parents se sont adressés à l'enfant chacun dans sa langue maternelle, le français pour le père, l'allemand pour la mère ${ }^{1}$. L'enfant est né et vit en France, et le français est ainsi la langue à laquelle il est le plus fréquemment exposé, l'exposition à l'allemand étant limitée aux interactions avec la mère et, deux ou trois fois par an, avec les membres de la famille lors de voyages en Allemagne.

2 Le corpus analysé ici est constitué de productions langagières précoces dans les deux langues concernées et couvre la période de 2 ans et 2 mois à 3 ans et 4 mois. Il fait l'objet d'une étude longitudinale plus large portant sur le développement de la compétence narrative entre 2 et 5 ans, qui implique entre autres la capacité de référer au temps.

Dans la présente étude nous tenterons de décrire les étapes d'émergence des différentes structures de subordination dans les deux langues, telles que nous avons pu les relever dans le corpus, pour ensuite déterminer leur contexte d'apparition et d'usage (conversation, récit conversationnel, récit à partir d'images). Dans ce contexte nous nous intéressons non seulement aux formes linguistiques et à leur chronologie d'apparition, mais également à leurs fonctions dans le discours de l'enfant. 


\section{Les étapes d'émergence des différentes structures de subordination et leur contexte d'apparition}

Pour déterminer les étapes d'émergence des différents schèmes de subordination, nous sommes partie des relateurs linguistiques tels qu'ils se présentent dans notre corpus, notamment des conjonctions subordonnantes, mais aussi des précurseurs des subordonnants comme les adverbes, pour ensuite analyser les fonctions exprimées. Comme point de départ nous nous servirons des productions dans ce qui était la langue faible à l'époque ${ }^{2}$, l'allemand, car l'émergence d'un grand nombre de structures de subordination temporelle correspond à un séjour de trois semaines en pays germanophone à l'âge de $2 ; 8^{3}$, qui a engendré une véritable explosion linguistique en ce qui concerne l'allemand. En effet, c'est à cette époque que l'autonomie de la langue faible s'est réellement forgée ${ }^{4}$.

5 En partant des conjonctions temporelles et causales dans nos données, nous notons la présence de plusieurs structures de subordonnées (temporelle et causale). Tout en décrivant les étapes de leur émergence, nous déterminerons leur contexte d'apparition et leur fréquence dans le discours de l'enfant et nous analyserons les fonctions exprimées ${ }^{5}$.

\section{Wenn ..., (dann) .../ quand, si..., ... + une forme verbale du présent ou du futur}

6 La subordination en wenn ...., (dann) ... / quand, si..., ... en position initiale est apparue vers $2 ; 8$ et environ 3 mois plus tôt en français, à $2 ; 5$.

7 Cette structure de subordination a fait son apparition sous forme d'une recherche de la conjonction, de la syntaxe et de la morphologie verbale adéquates. Ainsi en français, dans les premières productions de ce type, l'adverbe anaphorique après remplit la fonction de connecteur, avec des surgénéralisations occasionnelles où cet adverbe apparait à la fois dans la subordonnée et dans la principale. L'exemple 1 illustre le continuum qui existe entre structures paratactiques et hypotactiques dans le langage enfantin :

\begin{tabular}{|l|l|}
\hline 1 & Après tu faire mon bib(er)on après je va un bonbon ? $(2 ; 5)$ \\
\hline & <Quand tu auras fait mon biberon, j'aurai un bonbon ?>6 \\
\hline
\end{tabular}

8 Dans cet exemple on remarque également le tâtonnement de l'enfant quant à la morphologie verbale. A défaut du futur morphologique, qui apparaîtra chez notre sujet vers $2 ; 10$, l'enfant emploie l'infinitif dans la subordonnée, et essaie de réaliser une forme du futur périphrastique, l'auxiliaire fléchi marquant le prospectif et le verbe lexical restant implicite.

9 L'apparition de cette structure de subordination en allemand se fait vers $2 ; 8$ avec danach, qui dans la langue adulte est un adverbe temporel anaphorique, comme conjonction donc sur le même modèle qu'en français ${ }^{7}$-, et omission occasionnelle de l'auxiliaire fléchi des formes composées : 
Danach ich aufgestanden, ... < =wenn ich aufgestanden bin, ... $(2 ; 8)$

(Après je levé,... < = quand je serai levé,...>)

10 Mais les problèmes purement linguistiques concernant ces structures en allemand ont rapidement été résolus par l'enfant. Ainsi, deux ou trois semaines plus tard, la conjonction wenn, les temps verbaux et la syntaxe (forme verbale fléchie en position finale dans la subordonnée et inversion sujet/verbe dans la principale) sont en voie d'acquisition :

\begin{tabular}{|l|l|}
\hline 3 & Wenn ich aufgestanden bin, du gibst mir/gibst du mir ein Bonbon. $(2 ; 9)$ \\
\hline \hline & (Quand je suis levé, tu me donnes un bonbon.) \\
\hline 4 & Wenn ich wiederkomme, will ich die Birne essen. $(2 ; 9)$ \\
\hline \hline & (Quand je rentre, je veux manger la poire.) \\
\hline
\end{tabular}

11 Collings (1990:38) cite des études sur des enfants germanophones monolingues qui affirment que dès l'apparition de ces structures, les éléments verbaux fléchis apparaissent en position finale dans la subordonnée - conformément aux règles de placement du verbe dans la langue adulte - même si au début, il manque parfois la conjonction ${ }^{8}$. Dans nos données la conjonction n'est jamais absente, même si elle est au début parfois erronée, même si dans les toutes premières productions de ce genre coexistent des structures avec et sans auxiliaire fléchi. Mais comme chez Collings, notre sujet bilingue place la forme verbale fléchie en position finale dès l'apparition de ces constructions ${ }^{9}$.

Chez notre sujet l'acquisition de la subordination en allemand coïncide avec l'acquisition d'un certain nombre d'autres particularités de la syntaxe allemande, telles que notamment l'inversion sujet/verbe (fléchi) dans les interrogatives ainsi que dans les propositions assertives avec complément en position initiale ${ }^{10}$.

13 Cette acquisition semble coïncider avec celle de l'inversion de sujet/verbe (fléchi) dans la principale, et nos données suggèrent que l'inversion et le placement de la composante fléchie en deuxième position sont étroitement liés et commencent à être acquis simultanément.

14 Cette acquisition semble influer sur les formes linguistiques du même type en français, puisque quand apparaît à cette époque et remplace son précurseur après, et la subordination en quand se construira suivant le même schéma de distribution des tiroirs verbaux qu'en allemand. L'enfant va employer le présent ou le passé composé dans la subordonnée sur le modèle du Perfekt allemand, et le passé composé ou le futur périphrastique dans la principale :

Quand j'ai fini (de) faire dodo/quand je me réveille, tu me donnes un bonbon. $(2 ; 9)$ 
Je vais te montrer ce soir quand t'es arrivé de la cuisine.< = quand tu auras fini de faire la vaisselle> $(2 ; 11)$ avis, par le degré supérieur de complexité des formes linguistiques en français, où la subordination de ce type requiert des formes du futur morphologique : le futur antérieur dans la subordonnée et le futur simple dans la principale. En allemand, par contre, présent ou Perfekt dans la subordonnée et présent dans la principale suffisent pour former cette structure de subordination, où d'autres indices tels que les traits sémantiques de temporalité inhérents aux verbes, les adverbes temporels ou les syntagmes prépositionnels expriment la temporalité déictique. Cependant, c'est souvent le contexte situationnel qui permet à lui seul d'inférer les informations temporelles déictiques. Ainsi il semble que les temps verbaux allemands employés dans ce contexte seraient des temps relatifs, contrairement aux temps verbaux français, qui eux seraient des temps absolus ${ }^{11}$.

En ce qui concerne le futur morphologique, qui est requis pour cette structure de subordination en français, il faut attendre $2 ; 10 / 11$ pour voir apparaitre les premières formes du futur dans la proposition subordonnée :

\begin{tabular}{|l|l|}
\hline 7 & Quand tu reviendras, tu as les mains froides. $(2 ; 11)$ \\
\hline & et $3 ; 3$ pour l'émergence du futur dans les deux propositions : \\
\hline 8 & Quand je serai grand, j'aurai des playmobils. $(3 ; 3)$ \\
\hline
\end{tabular}

17 A cet âge, ces formes ne sont évidemment pas encore complètement acquises mais en voie d'acquisition. Ainsi à $3 ; 3$ coexistent des formes 'futur/présent' et 'futur/futur', de même qu'une surgénéralisation de la 3ème à la 2ème personne du singulier :

\begin{tabular}{|l|l|}
\hline 9 & Tu sais, papa, quand je serai grand, j'aurai ... (3;3) vs. \\
\hline 10 & Quand je sera grand, je l'aime <l' =la crème du lait>. (3;3) \\
\hline
\end{tabular}

Il en est de même pour l'allemand où nous avons observé des formes correctes dès $2 ; 8 / 9$ : la stabilisation des formes dans tous les contextes est un processus long.

Dans ce contexte, signalons que le pronom interrogatif wann (quand) fait son apparition vers $2 ; 10$ :

\begin{tabular}{|l|l|}
\hline 11 & Wann fährst du los ? Jetzt ? (2;10) \\
\hline \hline & (Quand pars-tu ? Maintenant ?) \\
\hline 12 & Wann kommt Antoinette wieder ? (2;10) \\
\hline \hline & (Quand revient Antoinette ?) \\
\hline
\end{tabular}


3 C'est quand qu'on va faire ça? $(3 ; 2)$

Les relations exprimées par la structure wenn ..., (dann).../quand, si...,...

Dans nos données la structure en wenn (quand, si) peut exprimer différentes relations entre les procès de la subordonnée et de la principale.

\section{a) Temporalité générique et concomitance entre le procès de la subordonnée et le procès de la principale}

21 Les subordinations en quand/wenn expriment une relation de concomitance entre le procès de la subordonnée et le procès de la principale. Cette relation temporelle est contextuelle, reconstructible par inférence. Signalons que, dans ce contexte, l'adverbe allemand dann (ex. 14 et 16) n'a pas la fonction temporelle APRÈS, mais une simple fonction cohésive.

La valeur temporelle pour les deux propositions est générique. Le temps verbal privilégié de ces structures est le présent dans chacune des deux langues et on y trouve des sujets génériques (man/on) et des adverbes génériques (immer/toujours).

\begin{tabular}{|c|c|}
\hline 14 & Wenn einer alleine losfährt, dann sagt man Gute Reise. $(2 ; 9)$ \\
\hline & (Quand quelqu'un part tout seul, on dit bon voyage.) \\
\hline & $\begin{array}{l}\text { Le plus souvent, l'expression du générique ou de l'habituel est associée à une relation de } \\
\text { condition ou de causalité : }\end{array}$ \\
\hline 15 & Da sind viele Leute. Wenn man vorbei will, muß man sagen pardon. $(2 ; 9)$ \\
\hline & (Là il y a beaucoup de monde. Si on veut passer, il faut dire pardon.) \\
\hline 16 & Wenn da der Löffel drin ist $<=$ im Topf>, dann kann man nicht richtig zumachen $<=$ den Deckel $>(2 ; 9)$ \\
\hline & (Si il y a la cuillère là-dedans < = dans la casserole $>$, on ne peut pas bien fermer $<=$ le couvercle $>$ ) \\
\hline 17 & Quand on mange pas, on aura faim. $(3 ; 2)$ \\
\hline 18 & Quand on fait pas la sieste, on est crevé, hein, le soir. $(3 ; 2)$ \\
\hline & Parfois il s'agit d'une pure relation temporelle de concomitance : \\
\hline 19 & Wenn die Dusche aus ist, hört man das. $(2 ; 9)$ \\
\hline & (Quand la douche s'arrête, ça s'entend <en entendant la douche s'arrêter>.) \\
\hline 20 & Wenn einer fragt $<=$ wie ich heiße>, sage ich Louis. $(2 ; 9)$ \\
\hline & (Quand quelqu'un me demande $<=$ comment je m'appelle $>$, je dis Louis.) \\
\hline
\end{tabular}


Wenn ich in der Badewanne sitz, kommt Papa immer und sagt mir Guten Tag. $(2 ; 11)$

(Quand je suis dans la baignoire, papa rentre toujours et me dit bonjour.)

\section{b) Postériorité déictique et concomitance entre le procès de la subordonnée et le procès de la principale}

Nous relevons dans nos données également des subordinations où les deux procès sont situés dans le futur. Dans les premières occurrences, le futur n'est pas exprimé explicitement (formes verbales du présent en allemand et en français), en français apparaissent ensuite les temps verbaux du futur: le futur périphrastique et le futur morphologique (cf. l'ex. 29). En allemand, où les temps verbaux du futur ne sont pas obligatoires pour référer au futur, le renvoi au futur reste implicite et peut être inféré du contexte (cf. l'ex. 30 par rapport à l'ex. 29). La relation temporelle entre le procès de la subordonnée et le procès de la principale est une relation de concomitance, reconstructible ici aussi le plus souvent à partir d'indices contextuels.

\begin{tabular}{|c|c|}
\hline 22 & Das weiß ich nicht. Das kann ich nicht sagen. Wenn Papa kommt, er erzählt das. $(2 ; 8)$ \\
\hline & (Je le sais pas. Je peux pas le dire. Quand papa rentre, il le raconte.) \\
\hline \multirow[t]{2}{*}{23} & Bestimmt hat Papa Schokolade. Wenn er kommt, frag ich ihn. $(2 ; 9)$ \\
\hline & (Papa a sûrement du chocolat. Quand il rentre, je vais lui demander.) \\
\hline \multirow[t]{2}{*}{24} & Wenn das $<=$ autocollant $>$ ab ist, sieht das $<=$ jouet $>$ wieder schön aus. $(2 ; 9)$ \\
\hline & (Quand ça sera parti, ça sera à nouveau joli.) \\
\hline \multirow[t]{2}{*}{25} & Wenn ich das aufgegessen habe, ich kriege noch was. $(2 ; 9)$ \\
\hline & (Quand j'ai fini de manger ça,j'en aurai encore.) \\
\hline 26 & Quand j'ai fini, tu me donnes mon dessert. $(2 ; 9)$ \\
\hline \multirow[t]{3}{*}{27} & $\begin{array}{l}\text { Wenn Papa mich aus dem Kindergarten abholt, bringt er mir die camionnette mit \Wie heißt das auf } \\
\text { deutsch? [Ad.: Lieferauto] Dann bringt er mir das Lieferauto mit. }(2 ; 11)\end{array}$ \\
\hline & (Quand papa vient me chercher à l'école, il m'apporte la camionnette...) \\
\hline & $\begin{array}{l}\text { A partir de } 3 ; 3 \text { nous trouvons des énoncés exprimant un enchaînement de deux relations de } \\
\text { postériorité: }\end{array}$ \\
\hline \multirow[t]{2}{*}{28} & $\begin{array}{l}\text { Wenn ich groß bin, kaufst du mir das, und wenn ich noch größer bin, kaufst du mir das. <en } \\
\text { montrant des jouets dans un magasin> }(3 ; 3)\end{array}$ \\
\hline & (Quand je serai grand, tu m'acheteras ça, et quand je serai encore plus grand, tu m'acheteras ça.) \\
\hline
\end{tabular}


Quand la $4 L$ sera cassée/ne marchera plus, papa va acheter une Peugeot verte, et quand la Peugeo sera cassée, il va acheter une camionnette. $(3 ; 3)$

30 Wenn die Quatrelle nicht mehr geht, kauft Papa ein(en) Peugeot, und wenn der Peugeot kaputt ist, kauft er eine Camionnette/ein Lieferauto. $(3 ; 3)$

(cf. l'exemple 29)

Il nous reste à signaler que dans tous les exemples cités il existe un lien "motivé » entre les procès des deux propositions, l'un des procès étant considéré comme "l'antécédent (logique ou causal), la raison ou encore la justification de l'autre » (Berthoud \& OtheninGirard, $1983: 189$ ).

\section{c) Condition}

En allemand, la conjonction wenn est à la fois un subordonnant temporel et conditionnel, ce qui fait qu'il est parfois difficile de savoir laquelle des deux notions prévaut dans les subordinations produites par notre sujet. Par contre, le locuteur français dispose d'un moyen distinct pour exprimer une relation conditionnelle entre deux procès en employant la conjonction si. La subordination en si est présente dans nos données, bien que de façon moins fréquente :

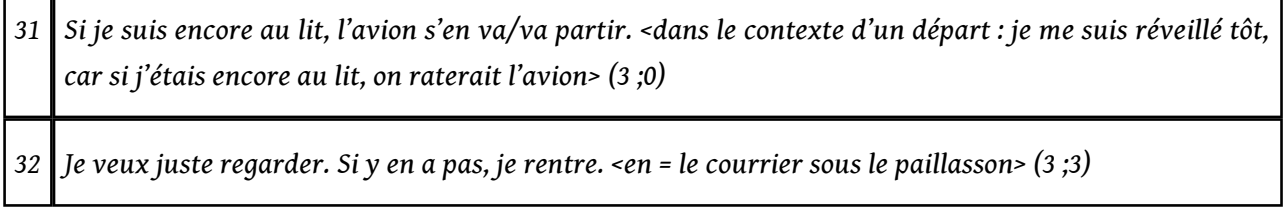

L'exemple 31 illustre la difficulté qu'a l'enfant d'exprimer l'irréel. L'enfant emploie le présent comme temps verbal. A défaut du conditionnel, c'est à nouveau le contexte qui permet d'inférer le caractère hypothétique des procès dont l'un se situe au présent et l'autre dans le futur, les deux n'étant pas assertés.

Dans l'exemple 32, les choses sont un peu plus faciles pour l'enfant, qui recourt au présent dans les deux propositions à valeur hypothétique.

L'emploi du présent est également caractéristique pour les premières subordinations conditionnelles en allemand. Nous renvoyons aux exemples 15 et 16. Ces exemples montrent cependant que la frontière entre relation temporelle et conditionnelle n'est pas toujours facile à établir.

\section{Contextes d'usage}

L'émergence et l'emploi de la subordination en wenn/quand, si se fait dans des conversations spontanées et semble constituer un enjeu important pour la maîtrise de son environnement. Pour ce qui est des énoncés à temporalité générique qui apparaissent dans des interactions libres et spontanées, l'enfant semble chercher des relations temporelles régulières dans sa vie quotidienne et dans le monde, qu'il verbalise ensuite en fonction de sa perception et de ses observations. Dans ce domaine, l'input langagier 
joue un rôle important, dans la mesure ou l'enfant organise les liens motivés entre les situations en s'appuyant largement sur les explications fournies par ses interlocuteurs.

Par ailleurs, cet enjeu pour l'enfant de s'orienter dans le monde au niveau temporel est manifesté par le fait que les subordinations sont souvent des explications ou des demandes de précisions de la part de l'enfant sur les relations temporelles entre événements à venir. L'enfant est à la recherche continuelle des relations de concomitance et de successivité et semble éprouver le besoin et l'envie de les expliciter lui-même.

Tout se passe comme si l'appropriation des notions de temps au niveau cognitif se faisait en partie à l'aide de la subordination qui déclenche des interactions avec l'entourage permettant à l'enfant de s'assurer de la compréhension d'adverbes et de propositions circonstancielles relevant entre autres des "cadres sociaux de la mémoire ${ }^{12}$ et d'exprimer lui-même ces relations temporelles. Ainsi un énoncé tel que quand je me réveille n'est dans le contexte qu'une stratégie de substitution d'expressions conventionnelles comme après la sieste ou demain matin. La subordination représente donc une paraphrase au moyen de laquelle l'enfant comble son déficit dans le domaine du temps social en attendant d'y avoir accès. Paradoxalement, pourrait-on dire, l'enfant préfère des structures hypotactiques, qui sont complexes, aux syntagmes nominaux, qui sont moins complexes. Mais du point de vue cognitif des expressions comme ce matin/ demain matin et même avant/ après la sieste sont plus abstraites pour l'enfant qu'une subordonnée telle que quand je me suis réveillé qui exprime une situation concrète vécue par l'enfant. Rappelons enfin que l'enfant a produit ces énoncés à l'âge de $2 ; 9$.

Dans nos données, nous constatons par ailleurs que l'enfant se trompe rarement de temps verbal, mais plutôt d'adverbe temporel. Dans un contexte situationnel donné, un énoncé comme demain matin j'ai pas fini mon lait renvoie au passé, et souvent c'est l'enfant luimême qui désambiguïse l'énoncé en rajoutant quand je me suis levé, c'est-à-dire ce matin. Ce fait nous semble suggérer que l'enfant maîtrise l'opposition passé/futur du point de vue cognitif, mais que ce sont notamment les expressions lexicales du temps conventionnel qui ne sont acquises que très progressivement. Les exemples de Sabeau-Jouannet (1977) vont dans le même sens.

Dans ce domaine, l'expérience individuelle joue un rôle primordial pour l'orientation temporelle. L'enfant choisit de construire ses subordonnées en wenn/quand avec comme repères privilégiés des moments revenant régulièrement dans sa vie quotidienne, comme en premier lieu le sommeil (jour et nuit) et les repas, mais aussi d'autres activités habituelles (le bain, le retour des parents, l'école) ou au contraire des activités sortant de l'habituel (voyages, fêtes, sorties), mais situées par rapport à ces situations familières à l'enfant.

En ce qui concerne les énoncés exprimant une relation de condition entre le procès de la principale et le procès de la subordonnée, ils ont également fait leur première apparition dans les conversations. L'enjeu pour l'enfant était de négocier le moment d'une activité désirée (sortie, etc.) ou (et surtout) celui de l'obtention d'une friandise ou autre récompense. Ces contextes sont donc évidemment étroitement liés aux situations familières à l'enfant et surtout aux événements qui lui tiennent particulièrement à cœur. 


\section{Als ..., .../quand..., .... + une forme verbale du passé}

35 La subordination temporelle en als/quand + une forme verbale du passé apparaît dans le langage de notre sujet pour la première fois à $2 ; 8$ pour devenir plus productive vers 3 ans. Elle spécifie les relations temporelles, de concomitance en général, entre le procès de la principale et le procès de la subordonnée. Ainsi ces énoncés servent à situer un événement passé par rapport à un autre ou à une situation-cadre révolue. Sur le plan linguistique, cette structure requiert la maîtrise des formes verbales du passé qui, chez notre sujet, sont le plus souvent le Perfekt et le prétérit pour l'allemand et le passé composé et l'imparfait pour le français :

\begin{tabular}{|c|c|}
\hline 33 & Als der Junge aufgestanden, er sieht : meine Hamster sind weg. $(2 ; 8)$ \\
\hline & (Quand le garçon levé, il voit: mes hamsters sont partis.) \\
\hline \multirow[t]{3}{*}{34} & Als wir aus der Kantine rausgegangen sind. $(3 ; 1)$ \\
\hline & (Quand on est sorti de la cantine.) \\
\hline & $\begin{array}{l}\text { <spécification temporelle en réponse à l'interrogation sur le cadre temporel: "Wann war das? } \\
\text { (C'était quand?)»> }\end{array}$ \\
\hline 35 & $\begin{array}{l}\text { Toi, tu travailles à Nanterre, où on est allé l'aut(re) fois quand j'étais un bébé. <= en fait juste } \\
\text { quelques mois auparavant> }(2 ; 11)\end{array}$ \\
\hline \multirow[t]{2}{*}{36} & $\begin{array}{l}\text { [Ad.: Wann hast du denn das gemacht?] Na, als du nich da warst. Und Papa hat mir nich geholfen. } \\
\text { Das hab ich alleine gemacht, als er die Küche gemacht hat/als er das Essen gemacht hat. }(3 ; 3)\end{array}$ \\
\hline & $\begin{array}{l}\text { ([Ad.: C'est quand que tu as fait ça ?] Ben, quand t'étais pas là. Et papa m'a pas aidé. Je l'ai fait tout } \\
\text { seul quand il a fait la cuisine.) }\end{array}$ \\
\hline 37 & Papa, quand j'étais à Montpellier, qu'est-ce que t'avais fait à Paris ? $(3 ; 3)$ \\
\hline 38 & Où [tete] garé quand t'es allé chez Annette? $(3 ; 3)$ \\
\hline
\end{tabular}

Dès l'apparition du plus-que-parfait en français, l'enfant le surgénéralise à des contextes qui requièrent le passé composé, et nous le trouvons occasionnellement dans les subordinations en quand exprimant des événements au passé sans qu'il y ait une relation d'antériorité entre les procès de la principale et de la subordonnée (cf. l'ex. 37) .

\section{Contextes d'usage}

La structure en als/quand + forme verbale au passé apparaît surtout dans des mini-récits spontanés. Elle est un moyen important pour situer temporellement l'événement-clé dont l'enfant prend l'initiative de faire un récit par rapport à une situation-cadre ou à d'autres événements, le plus souvent rituels et donc connus des interlocuteurs. Ces précisions temporelles ont d'abord été sollicitées par l'interlocuteur pour être petit à petit données 
par l'enfant sans qu'on le lui demande, et cela souvent en début de récit, à la fois servant de marqueur de prise de parole et d'indication temporelle :

39 Tu sais, quand on était chez Mamet, ...

38 L'étayage de l'entourage a ainsi amené l'enfant à prendre l'habitude de commencer un récit d'expérience personnelle en spécifiant le cadre temporel avant toute autre chose et en le donnant en topique de l'énoncé initial du récit.

Les structures en wenn/ quand, si et als/ quand sont les plus fréquentes dans nos données, mais nous y trouvons des occurrences de quelques autres structures temporelles.

\section{3. ... (solange) ..., bis .../(... jusqu'à ce que...)}

Il s'agit de la subordination (solange) ... bis en allemand qui apparaît de même que les autres à $2 ; 8$. Contrairement aux constructions analysées jusqu'ici où la subordonnée et le subordonnant se trouvaient, à quelques exceptions près, en position initiale, c'est la principale de la structure en ... (solange) ..., bis... qui se trouve en position initiale, suivie par la subordonnée.

\begin{tabular}{|l|l|}
\hline 40 & Dann wart ich, bis die Autos wegfahren. Ich muß noch warten, bis die Autos wegfahren. $(2 ; 8)$ \\
\hline & $\begin{array}{l}\text { (Puis j'attends jusqu'à ce que les voitures partent. Je dois encore attendre jusqu'à ce que les voitures } \\
\text { partent.) }\end{array}$ \\
\hline 41 & Dann hab ich solange auf der Bank gewartet, bis du kommst. (2;9) \\
\hline \hline & (Aprèsj'ai attendu sur le banc jusqu'à ce que tu viennes.) \\
\hline
\end{tabular}

41 La principale contient un trait temporel de la durée qui peut être renforcée par l'adverbe solange. La subordonnée explicite la borne de droite de l'intervalle temporel donné dans la principale, dans nos exemples de l'attente.

42 Or dans nos données nous ne trouvons pas d'équivalents français du type jusqu'à ce que. Cette forme étant beaucoup plus complexe à manier en français (subjonctif) qu'en allemand, l'enfant ne renonce pas à exprimer ce contenu, mais opte pour des moyens linguistiques moins complexes à sa disposition, notamment la complétive :

42 J'ai attendu que tu viens. (ou bien tout simplement : je t'ai attendu)

43 Un autre facteur susceptible d'expliquer la relative disparité de l'acquisition de structures en (solange) ... bis / jusqu'à ce que... est probablement la fréquence de ces structures en allemand et en français parlé, et notamment dans le langage auquel sont exposé les petits germanophones et les petits francophones. Alors que le locuteur allemand n'a pas de raison d'éviter cette forme, l'adulte français tend à opter pour d'autres structures, notamment lorsqu'il s'adresse à des petits enfants. 


\section{Contextes d'usage}

44 Nous avons trouvé les quelques occurrences de la structure (solange) ... bis ... dans des situations de jeu autonome et symbolique ainsi que dans des mini-récits conversationnels.

\section{4. bevor (avant que)}

En allemand nous notons la présence d'une tentative de l'enfant pour utiliser la structure en bevor, mais l'enfant y renonce aussitôt, la remplaçant par une structure prépositionnelle :

\begin{tabular}{|c|c|}
\hline 43 & $\begin{array}{l}\text { Ad. : Sag mal Louis, als dir das Unglück passiert ist + mit Hamid + war das vorm Mittagessen, bevor } \\
\text { ihr in die Kantine gegangen seid oder bevor Papa gekommen ist? }\end{array}$ \\
\hline & $\begin{array}{l}\text { (Ad.: Dis Louis quand t'est arrivé le malheur avec Hamid + c'était avant le déjeuner avant que vous } \\
\text { alliez à la cantine? ou avant que papa vienne te chercher?) }\end{array}$ \\
\hline & $L:$ nee (non) \\
\hline & Ad.: Wann war das? (Cétait quand?) \\
\hline & $L:$ Vor wir gegessen / vor der Kantine vor \(Avant nous mangé / avant la cantine avant $\backslash$ ) \\
\hline & Ad. : Vor der Kantine ah ja (Ad. : avant la cantine ah d'accord) \\
\hline & $\begin{array}{l}\text { L: Nee, weißte wann ? ++W/++ Als wir aus der Kantine rausgegangen sind. (Non, tu sais quand? } ?^{++++} \\
\text {Quand on est sorti de la cantine.) }(3 ; 1)\end{array}$ \\
\hline
\end{tabular}

Il s'agit d'une imitation directe, et l'absence d'autres occurrences de cette structure dans la tranche d'âge analysée ici semble être en relation avec sa complexité d'une part, et la faible fréquence dans l'input langagier d'autre part. L'expression hypotactique de l'inversion de la chronologie semble encore poser problème à notre sujet. En effet, pour exprimer cette relation, le locuteur peut également choisir la forme prépositionnelle (vor/ avant) + nom, d'autant plus qu'en allemand on peut former un nom à partir de nombreux verbes en leur attribuant l'article neutre (das --> vor dem/vorm Waschen, Essen, Schlafengehen, Zähneputzen, etc. --> verbes substantivés)

En français, l'enfant va recourir à la forme avant de + infinitif:

44

Avant de partir on a pas mis le répondeur. Après de partir on a pas mis le répondeur. On est parti sans répondeur allumé. $(3 ; 2)$

45 Tu sais, à la ludothèque, avant de partir j'ai rangé. L'aut(re) fois je voulais pas. $(3 ; 2)$

Or, même dans ces exemples français où l'utilisation de l'infinitif du verbe précédé par la préposition de devrait être plus facilement maniable par l'enfant que la structure 
allemande en bevor/avant que, qui requiert une morphologie verbale et une syntaxe complexes, nous notons trois tentatives de formulation successives pour exprimer une relation temporelle inversée (ex. 42). L'hésitation entre avant de partir et après de partir et la résolution du problème par une toute autre formulation (sans répondeur allumé) semblent liées à la représentation cognitive de l'inversion de la chronologie des événements.

Nous nous proposons maintenant de faire un retour en arrière pour analyser la fonction de certaines formes linguistiques présentes dans les précurseurs de la subordination temporelle et pour déterminer leur rôle dans la construction progressive de la subordination et d'autres structures linguistiques. La place la plus importante, dans la recherche par l'enfant du mot ou de la construction adéquats, revient à l'adverbe anaphorique danach/après, et certains des phénomènes observés ont pu être attestés dans les deux langues.

\section{Danach (après) - mot plurifonctionnel}

Pendant une période brève et transitoire de l'acquisition du langage par notre sujet, qui se situe aux alentours de $2 ; 8$ et qui correspond à la période d'apparition de la compétence germanophone, l'adverbe anaphorique allemand danach comblait bien des lacunes dans la construction des énoncés par l'enfant. Ainsi danach a rempli plusieurs fonctions pendant quelques semaines :

a) la fonction de l'anaphorique temporel de postériorité dann, danach (après, ensuite)

\begin{tabular}{|l|l|}
46 & $\begin{array}{l}\text { Ich geh kurz runter ... danach je komme wieder hoch }+ \text { ich komm in bett rein und danach ich schlafe } \\
\text { noch danach }(2 ; 8)\end{array}$ \\
\hline & (Je vais vite descendre ... après je remonte + je viens au lit et après je dors encore après)
\end{tabular}

50 La première fonction de danach correspond ici à ce qui est également sa fonction dans le langage adulte, celle d'adverbe temporel anaphorique qui permet d'exprimer la succession des procès dans leur chronologie.

b) la fonction de l'anaphorique temporel d'antériorité vorher, davor (avant)

\begin{tabular}{|l|l|}
47 & Jetzt sind wir in Paris, und danach wir waren in H. $(2 ; 8)$ \\
\hline & (Maintenant on est à Paris et après on était à H.)
\end{tabular}

51 Dans l'exemple 47, la fonction de danach correspond à l'exact contraire de sa fonction première. Nous ne relevons qu'occasionnellement dans nos données des occurrences d'un emploi de danach avec cette fonction, lorsque l'enfant l'utilise à la place de l'adverbe temporel d'antériorité vorher, davor (avant). Cet exemple soulève à nouveau le problème de l'inversion chronologique que l'enfant met du temps à exprimer par des moyens appropriés. Quand l'enfant commence à construire des énoncés n'exprimant pas les procès dans l'ordre dans lequel ils se sont déroulés, le choix de l'adverbe temporel lui pose problème. Or, dans ce cas non plus, l'enfant ne se trompe pas de temps verbal : dans 
notre exemple, présent dans la première proposition et passé dans la deuxième. Visiblement c'est encore une fois la représentation cognitive de l'inversion de la chronologie qui est en cause, et le choix erroné de l'adverbe temporel nous semble en constituer une trace pertinente.

c) la fonction de la conjonction conditionnelle sonst (sinon) exprimant le résultat hypothétique du non-accomplissement d'une condition

\begin{tabular}{|l|l|}
\hline 48 & Ich muß die Kapuze aufsetzen, danach wird mein Kopf naß. (2;8) \\
\hline & (Il faut que je mette la capuche, après ma tête sera mouillée.) \\
\hline 49 & Ich muß das mal machen, danach geht das nich. $(2 ; 9)$ \\
\hline & (Je dois faire ça, après ça marche pas.) \\
\hline
\end{tabular}

52 Il nous semble que cet emploi chez notre sujet est influencé par la langue forte dans la mesure où en français parlé après (danach) peut remplacer sinon dans certains contextes. Ainsi des énoncés tels que Ne monte pas sur l'arbre, après tu vas tomber sont assez fréquents dans le langage adulte adressé aux enfants. Or notre sujet fait une surgénéralisation de cette construction; on ne peut effectivement pas non plus dire en français Il faut que je mette la capuche, après ma tête sera mouillée. La construction en après suit uniquement les injonctions négatives en français familier, requiert donc l'impératif et la négation, alors que la construction en sonst/sinon exprime une situation hypothétique résultant du nonaccomplissement d'une condition.

53 De plus, on peut se demander si l'enfant emploie un adverbe temporel parce qu'il perçoit dans cette relation conditionnelle la dimension temporelle de postériorité, telle qu'elle se trouve exprimée en français familier.

L'enfant lui-même produisait quelque temps auparavant des énoncés avec après tels que

\begin{tabular}{|c|c|}
\hline 50 & attends il faut laver $<=$ enlever $>$ le mann + noch et après ça geht + après ça geht pas + danach $(2 ; 6)$ \\
\hline & (attends il faut enlever le bonhomme + encore et après ça marche + après ça marche pas + après) \\
\hline & $\begin{array}{l}\text { où le premier après exprime une relation temporelle de postériorité et où le second remplit la fonction } \\
\text { de sonst/sinon, donc un résultat hypothétique. }\end{array}$ \\
\hline & $\begin{array}{l}\text { Signalons enfin les premières occurrences dans nos données d'énoncés avec sonst/sinon, dont } \\
\text { l'apparition se situe aux alentours de trois ans: }\end{array}$ \\
\hline 51 & Kannst du mich mal ranrücken? Sonst fällt der noch runter. $(3 ; 1)$ \\
\hline & (Tu peux me rapprocher < de la table> ? Sinon il va tomber <il = une peluche>.) \\
\hline 52 & Und ich steck die nich in $n$ Mund, sonst verschluck ich die. $(3 ; 2)$ \\
\hline & (Et je ne les mets pas à la bouche, sinon je les avale <les = des trombones>.) \\
\hline
\end{tabular}


[Ad. : Arrête.] Sinon ça va mal aller. $(3 ; 2)$

d) précurseur de la conjonction temporelle wenn (quand) dans une subordonnée exprimant un procès au futur

\begin{tabular}{|l|l|}
\hline 54 & Danach ich aufgestanden (bin), danach wir fahren zu P. $(2 ; 8)$ \\
\hline \hline & (Après je suis levé, après on va chez P.)
\end{tabular}

e) précurseur de la conjonction temporelle als (quand, lorsque) dans une subordonnée exprimant un procès au passé

\begin{tabular}{|l|l|}
\hline 55 & Danach ich klein war, ... (2;8) \\
\hline & (Après j'étais petit,...) \\
\hline 56 & Danach ich ein Baby war, ... $(2 ; 8)$ \\
\hline & (Aprèsj'étais un bébé,...) \\
\hline
\end{tabular}

57 Dans ces exemples danach remplit les fonctions de la conjonction temporelle als et la morphologie verbale semble à nouveau indiquer que le procès exprimé est représenté par l'enfant au passé, mais que la conjonction exprimant cette relation n'est pas encore acquise.

\section{5. parce que [paske]/weil}

Nous analyserons à présent les structures en [paske], qui sont à la fois les plus fréquentes et les plus précoces dans nos données.

La subordination causale en parce que [pak]->[pask(e)] apparaît dès l'âge de $2 ; 3$ en français et peu avant l'interrogatif pourquoi, qui est aussitôt intégré dans des questions telles que pourquoi il pleure? pourquoi tu t'es fait mal ?, etc. Il faudra attendre $2 ; 5 / 6$ pour trouver l'interrogatif allemand correspondant warum, wieso isolément et $2 ; 8$ pour voir émerger la conjonction weil en allemand et wieso dans des propositions interrogatives.

Ce contexte nous permet de faire une petite parenthèse pour illustrer la genèse de la langue faible dans ce domaine. Lorsque l'enfant commence à utiliser l'interrogatif allemand wieso isolément, il continue à avoir recours à l'interrogatif français et à la syntaxe française pour former des questions plus précises :

\begin{tabular}{|l|l|}
\hline 57 & Wieso ? (Pourquoi ?) \\
\hline 58 & Pourquoi is sein bein da ? $(2 ; 8)$ \\
\hline & (Pourquoi est sa jambe là $?<=$ allemand standard $>)$ \\
\hline
\end{tabular}




\begin{tabular}{|l|l|}
\hline & coexistent avec \\
\hline 59 & Wieso il est runtergefallen ? - Wieso il a einen Schreck gekriegt ? $(2 ; 8)$ \\
\hline & (Pourquoi il est tombé ? - Pourquoi il a pris peur ?) \\
\hline
\end{tabular}

61 Tout se passe comme si, pendant cette phase transitoire, l'enfant devait choisir entre une construction avec l'interrogatif français et l'inversion sujet/verbe des éléments allemands (ex. 58) et une construction avec l'interrogatif allemand, des éléments français et la syntaxe française (ex. 59).

62 La subordination causale en français est apparue avec la conjonction parce que, et elle semble exprimer dès son apparition à la fois une causalité du monde physique et une causalité intentionnelle ${ }^{13}$. Elle est donc en relation avec des phénomènes du monde physique qui sont parfois à l'origine de l'intention des actants. Ainsi les exemples les plus précoces associent les deux catégories de causalité :

60

On va les ranger les voitures [paske] ça tombe par terre dans le train. $(2 ; 3)$

61 I faut mett(re) le bonnet [pask] i fait froid dehors. $(2 ; 3)$

63 Comme les premiers énoncés de ce genre soit expriment une causalité pour des événements génériques, soit renvoient à la situation d'énonciation, cette structure de subordination fait son apparition dans des énoncés ayant le présent comme temps verbal, pour ensuite se diversifier morphologiquement au niveau temporel. Ainsi le procès exprimé dans la subordonnée peut se situer à un moment antérieur ou postérieur au moment de la parole. L'enfant marque cet ancrage progressivement par les temps verbaux du passé et du futur, notamment le passé composé et l'imparfait pour le passé et le futur périphrastique pour le futur :

\begin{tabular}{|c|c|}
\hline 62 & Si, je pleure paske papa est pa(r)ti au travail< = j'ai pleuré> $(2 ; 4)$ \\
\hline 63 & T'attends là. T'attends? Paske je vais ranger la voiture. $(2 ; 7)$ \\
\hline \multirow[t]{2}{*}{64} & $\begin{array}{l}\text { Paske je voulais in dein Bett rein(s)teigen [Ad. : Warum denn ?] Pask/ Weil j'ai j'ai runtergesteigen } \\
\text { aus mon/mein Bett. }(2 ; 8)\end{array}$ \\
\hline & (Paske je voulais venir dans ton lit. [ Ad.: Mais pourquoi ?] Paske je suis descendu de mon lit.) \\
\hline \multirow[t]{2}{*}{65} & Papa i m'a gehalten paske je noch ein bißchen $k(l)$ ein. $(2 ; 8)$ \\
\hline & (Papa i m'a tenu paske je encore un peu petit.) \\
\hline & $\begin{array}{l}\text { L'acquisition de la subordination de causalité en allemand se met progressivement en place } \\
\text { vers } 2 ; 8 \text { et l'enfant ne réalise pas tout de suite le placement de la forme verbale fléchie en } \\
\text { position finale }{ }^{14} \text { : }\end{array}$ \\
\hline
\end{tabular}




\begin{tabular}{|l|l|}
\hline 66 & Na weil ich will noch spielen. $(2 ; 8)$ \\
\hline & (Ben parce que je veux encore jouer.) \\
\hline \hline & $\begin{array}{l}\text { La forme vermand standard : weil ich noch spielen will }> \\
\text { temps que dans d'autres contextes obligatoires (cf. ci-dessus) : }\end{array}$ \\
\hline 67 & Fahr ganz langsam, weil die Straße so glatt is. ( $2 ; 8)$ \\
\hline & (Roule tout doucement, parce que ça glisse sur la route.) \\
\hline
\end{tabular}

Mais par la suite, le placement de la forme verbale fléchie en position finale dans ce contexte s'avère être moins stable que dans d'autres. Ainsi coexistent dans le langage de l'enfant des subordonnées en weil/na weil avec la forme verbale fléchie aussi bien en position finale qu'en deuxième position. A notre avis, plusieurs facteurs sont à l'origine de cette variation: d'une part, on trouve dans la langue courante des subordonnées en weil où la forme verbale fléchie n'est pas en position finale ${ }^{15}$, mais en deuxième position comme dans une proposition indépendante. D'autre part, cet emploi est fréquent dans la langue à laquelle notre enfant est exposé, ne serait-ce que parce que ses interlocuteurs hésitent parfois un moment avant de répondre aux nombreux pourquoi posés par l'enfant. Cette hésitation engendre une pause « de réflexion » après la conjonction qui sera suivie d'une proposition indépendante :

Wieso fährt der Mann los ? + Na weil ++ der fährt vielleicht nach Hause. (S) (V)

(Pourquoi le monsieur s'en va ? + Ben parce que ++ il rentre peut-être...)

Un troisième facteur pourrait être l'influence du français, où ce schème de subordination ne nécessite aucun changement au niveau syntaxique par rapport à l'ordre des éléments dans une proposition indépendante. Cependant, ce facteur ne nous parait pas prépondérant, vu l'acquisition des règles de placement de verbe en l'allemand du même ordre dans d'autres contextes obligatoires, acquisition qui s'est faite rapidement et sans problèmes majeurs.

66 La réponse de l'enfant à des pourquoi, qu'il n'est pas en mesure de donner, correspondra au modèle du français.

69 Na weil. $([$ Paske.] $]$ < allemand standard : Darum.>

\section{Contextes d'usage}

67 Les structures en paske ont fait leur apparition dans les conversations libres et les situations de jeu autonome pour ensuite être employées dans les récits à partir d'images. 

phénomènes du monde physique, mais également sur les motivations des actants. Ainsi, dans notre corpus, les deux catégories établies entre autres par Cordier (1994) sont difficiles à démêler et semblent se développer simultanément. Lors des toutes premières productions de subordonnées causales en paske, notre sujet s'appuie d'ailleurs en partie sur l'étayage de l'entourage, les interactions adulte-enfant permettant à l'enfant d'acquérir la notion de causalité dans des contextes qui lui sont familiers. L'exemple 61, produit spontanément, en est une bonne illustration. Cependant, quelques semaines plus tard l'enfant raisonnera de façon de plus en plus autonome sans attendre les informations fournies par les adultes.

qui concerne l'expression des relations causales dans les récits, notamment à partir d'images, l'appropriation de l'histoire par l'enfant semble en grande partie reposer sur la compréhension progressive des relations cause-effet, à la fois dans le domaine du monde physique et celui de l'intentionnalité. L'étayage que l'enfant sollicite de la part de ses interlocuteurs s'explique à notre avis par le fait que l'enfant est parfois confronté à des événements et à des modes d'action qui ne lui sont pas familiers et dont il ne peut pas puiser les relations causales dans son expérience personnelle.

Quant aux situations de jeu autonome, l'expression des relations causales sert essentiellement à prêter une vie psychologique, et plus précisément une motivation, aux actants dont les rôles sont symboliquement joués par notre sujet.

\section{7. damit .../pour que ... vs. um ... zu .../pour...}

71 Signalons enfin l'existence dans nos données d'autres constructions complexes comme celles exprimant le but. Notre sujet ne dissociait pas clairement les structures de subordination en damit et pour que des infinitives en um ... zu et pour lors de leur émergence. C'est pourquoi nous traitons cette étape d'acquisition globalement.

L'enfant avait à sa disposition en français la forme pour + infinitif dès l'âge de $2 ; 2$ et pouvait ainsi exprimer des relations de but avec infinitif seul :

\begin{tabular}{|l|l|}
\hline 70 & pour manger, pour accrocher, etc. \\
\hline & et rapidement avec infinitif + syntagme nominal: \\
\hline 71 & pour accrocher la remo(r)que \\
\hline $\begin{array}{l}\text { En allemand, les précurseurs de la subordination exprimant le but apparaissent vers } 2 ; 8, \text { et l'enfant a } \\
\text { recours à l'élément zu des infinitives allemandes: }\end{array}$ \\
\hline 72 & $z u$ alle beide spielen/um alle beide spielen $(2 ; 8)$ \\
\hline 73 & Ich hole meine Autos zum mit dir spielen. $(2 ; 8)$ \\
\hline \hline & (je vais chercher mes voitures pour jouer avec toi.) \\
\hline
\end{tabular}


zum Papa das geben $(3 ; 4)$

(pour donner ça à papa) éléments um, qui se place en position initiale de la subordonnée, et $z u$, qui se place avant l'infinitif, donc en avant-dernière position. La forme zum (préposition+article) n'est admise qu'avec un verbe substantivé (zum Spielen).

On peut d'ailleurs se demander si ces tentatives de construction avec zu/zum ne suivraient pas le modèle français de l'infinitive avec pour où la syntaxe est moins complexe.

Cette hypothèse se trouve d'une certaine façon confirmée dans nos données, où nous constatons vers 3 ans la présence de la préposition für (cf. pour) qui remplit la fonction de damit (cf. pour que en français) :

\begin{tabular}{|l|l|}
\hline 75 & Da mach ich den Zaun hin, für keine Hunde reinkommen. $(3 ; 0)$ \\
\hline \hline & (Là, je mets la clôture pour que les chiens n'entrent pas.) \\
\hline
\end{tabular}
cognitif, du moins pour les situations familières à l'enfant et pour les contenus que l'enfant prend lui-même l'initiative de verbaliser, mais où ce sont les formes linguistiques, relativement complexes et peu fréquentes dans le langage de l'entourage, qui posent problème. Or, même si la notion de la relation de but semble acquise, il reste le problème linguistique à résoudre par l'enfant: il tâtonne dans le domaine des connecteurs et de la syntaxe, dans la mesure où dans une infinitive le verbe n'est pas fléchi et où il y a coréférence entre actants des deux propositions. A l'inverse, une subordonnée de but en damit contient une forme verbale fléchie et la coréférence entre actants est possible, mais ni obligatoire ni implicite. C'est là qu'à notre avis réside la difficulté majeure à acquérir ces formes linguistiques.

En français aussi, s'ajoute au problème de représentation cognitive la complexité de la construction linguistique, dans la mesure où la conjonction de but pour que requiert le subjonctif, qui dans nos données apparaît à $3 ; 2$. Cette forme fait son apparition dans tout un ensemble de contextes obligatoires qui feront l'objet de véritables gammes sur le subjonctif, tels que : i faut que, j'ai peur que, je veux que.

\section{la conjonction pour que :}

$$
\begin{array}{|l|l|}
\hline 76 & \text { C'est pour je répare le garage. }(3 ; 2) \\
\hline 77 & \text { Je fais le lit pour qu'i dort. Il fait déjà nuit. }(3 ; 2) \\
\hline
\end{array}
$$


C'est vers $3 ; 4$ qu'apparaissent les premières formes irrégulières correctement formeés telles que: j'ai peur que je vous perde, $i$ faut que j'aille..., $i$ faut que tu fasses..., qui ont évidemment été précédées par leurs formes indicatives.

\section{Conclusion} dans nos données provenant de conversations libres, et si elles sont présentes à un certain degré dans des récits spontanés d'expérience personnelle où l'enfant prend luimême l'initiative de raconter des événements vécus, il n'en est pas tout à fait de même pour les récits faits à partir d'un livre d'image à la demande d'un interlocuteur. On peut donc se demander quelle est la fonction de la subordination temporelle dans le langage enfantin, et quelle est la relation entre développement cognitif et linguistique dans ce domaine, dans la mesure où la capacité narrative ne suppose pas uniquement une capacité linguistique, mais aussi des capacités discursives et communicationnelles de même que la conceptualisation des procès à mettre en mots et leur organisation en une structure hiérarchisée.

81 Il nous semble que, même si les formes acquises permettent à l'enfant d'exprimer antériorité et postériorité par rapport au moment de la parole, et de mettre en relation temporelle deux événements du passé ou du futur ${ }^{16}$, on n'avait pas à attendre l'émergence de formes subordonnées pour trouver des traces d'un détachement du ici et maintenant dans le langage enfantin. En effet, dès l'apparition de la morphologie verbale du passé et du futur et des adverbes et locutions prépositionnelles, l'enfant a la possibilité d'exprimer ces notions par des structures paratactiques comme la sériation tout en se servant de ces adverbes et expressions adverbiales de temps ou même de lieu, qui spécifient l'intervalle du moment en question explicitement.

'acquisition plus tardive des structures subordonnées semble d'une part être liée à la complexité des formes linguistiques, et d'autre part aux fonctions de la subordination au niveau discursif, dont l'acquisition et très graduelle, permettant par exemple à l'enfant de placer un événement en topique de l'énoncé, tandis qu'un autre événement sera en focus.

Si l'enfant commence vers trois ans à constituer l'arrière-plan de ses mini-récits spontanés à l'aide de la subordination pour fournir les indications temporelles, nous n'en trouvons pratiquement pas de trace dans les récits à partir d'images, où il y a quasiabsence de subordination temporelle. L'enfant, qui a tendance à décrire les images les unes après les autres sans mettre en mots toutes les informations données dans les images, exprime les relations temporelles par d'autres moyens : la succession par des connecteurs comme et, da (alors), alors et des adverbes temporels comme après et la simultanéité également par et. S'il y a dans notre corpus de récits à partir d'images des occurrences d'autres types de subordination, telles que les relatives et les complétives en premier lieu, nous notons les premières apparitions de la subordination temporelle dans ces récits entre trois ans et demi et quatre ans.

Cette absence de subordination temporelle dans les récits précoces est confirmée par d'autres travaux. Dubosq-Sénémaud (1997), qui a entrepris de déterminer les indices linguistiques d'un âge langagier à partir de récits sur images de plus de 200 enfants francophones d'âge différents, date l'âge de l'émergence des premières subordinations temporelles dans le récit à $4 ; 9^{17}$. Kern (1997), qui a également analysé les productions 
narratives d'enfants francophones, observe deux premières occurrences de subordinations temporelles dans la tranche d'âge de $3 / 4$ ans (les deux sujets concernés ont respectivement l'âge de $4 ; 4$ et $4 ; 8$, la tranche d'âge étant constituée de 14 sujets) et 17 occurrences dans la tranche d'âge de 5 ans (20 sujets dans cette tranche d'âge ${ }^{18}$. Bocaz (1989), qui a étudié l'expression de la temporalité dans des récits sur images produits par des enfants hispanophones ( $\mathrm{du}$ Chili et d'Argentine), relève quatre premières occurrences de subordinations temporelles dans la tranche d'âge de 3 ans (qui va de $3 ; 2$ à $3 ; 8$ ).

Curieusement, dans les récits précoces $(2 ; 8 / 2 ; 9)$ à partir de livres d'images tel que Frog, Where Are You ${ }^{19}$, nous notons à plusieurs reprises l'expression de relations de causalité, concernant notamment la cause externe de certains événements et les motivations des actants, relevant donc et de la causalité du monde physique et de l'intentionnalité.

Notre sujet n'étant pas placé dans un cadre purement expérimental pour cette tâche, mais racontant l'histoire à l'un de ses parents au foyer familial, il est difficile d'éviter un certain degré d'étayage. Ainsi, dans les premières versions (à $2 ; 8 / 9$ ) nous nous trouvons face à une co-construction du récit sous forme d'une interaction questions-réponses, et nous observons surtout dans ces premières tentatives de récits à partir d'images une focalisation de la part de l'enfant sur le pourquoi des événements et des motivations des actants $^{20}$. D'une part, la présence fréquente du pronom interrogatif pourquoi dans ces récits conversationnels semble être une trace du travail de l'appropriation de l'histoire par l'enfant à un très jeune âge, d'autre part, le très jeune enfant semble s'identifier aux actants, notamment au protagoniste de l'histoire, le petit garçon. Pour ce travail de compréhension et d'appropriation d'une histoire, l'enfant part de ses expériences individuelles pour aboutir à une représentation cohérente, et lorsque son expérience personnelle fait défaut, il va demander pourquoi tel événement a lieu ou pourquoi tel actant agit de telle façon.

De plus, on se demande s'il n'y a pas un décalage entre la représentation de l'histoire et sa mise en mots, le problème pour l'enfant n'étant pas seulement la construction d'une vision globale, c'est-à-dire d'une histoire ayant un début, une fin et organisée en épisodes temporo-causaux, mais l'organisation verbale de cette représentation. Cette organisation demande à l'enfant de se représenter les besoins en informations de type référentiel de la part du destinataire pour assurer la cohésion du récit, et de satisfaire un besoin communicatif, à savoir anticiper la compréhension par l'interlocuteur. Dans notre cas, cette tâche est d'autant plus difficile pour l'enfant que dans une situation familiale il peut supposer que l'histoire est connue du destinataire. L'investissement personnel de la part de l'enfant sera donc moindre que dans les récits spontanés, où il y a un véritable besoin communicatif.

Un deuxième facteur pouvant expliquer l'absence de subordination temporelle dans les récits très précoces à partir d'images semble être le fait que l'histoire n'ait pas été vécue par l'enfant. En effet, les récits d'événements vécus par l'enfant semblent davantage susceptibles d'engendrer des indications concernant tous les domaines de référence et parmi eux des indications temporelles. Les situations étant vécues par l'enfant et donc non fictives, le rappel de leurs circonstances semble faciliter l'organisation du récit. Pour ce qui est des récits à partir d'images, c'est plutôt l'enfant lui-même, surtout lors d'une première familiarisation avec une histoire, qui se place en position de demandeur de précisions et notamment d'explications sur les relations causales. 
89 Lesuisition des notions de causalité et de temporalité semble donc reposer sur deux grands facteurs : elle se fait par le biais du vécu de l'enfant d'une part, et par le langage comme outil d'appropriation de catégories représentatives de l'autre.

\section{BIBLIOGRAPHIE}

BAMBERG, M. (1994), “Development of Linguistic Forms: German”, chapitre IIIB de Berman, R. A. \& Slobin, D. I. Different Ways of Relating Events in Narrative: A Crosslinguistic Developmental Study, Hillsdale, NJ, Erlbaum.

BERMAN, R. A. \& SLOBIN, D. I. (1994), Different Ways of Relating Events in Narrative: A Crosslinguistic Developmental Study, Hillsdale, NJ, Erlbaum.

BERTHOUD, I. \& OTHENIN-GIRARD, C. (1983) : «L'étude génétique des subordonnées : propositions méthodologiques ", chapitre 11 de Bronckart, J.-P., Kail, M. \& Noizet, G. Psycholinguistique de l'enfant, Neuchâtel, Paris, Delachaux \& Niestlé.

BOCAZ, A. (1989), “Los marcadores de expresión de la simultaneidad en el desarrollo de estructuras sintácticas y textuales complejas”, Revista de Lingüística Teórica y Aplicada, 27, Concepción (Chili), 5-22.

COLLINGS, A. (1990), “The acquisition of morphology and syntax in German child language”, chapitre 2 de Meisel, J. M. (éd.) Two First Languages - Early Grammatical Development in Bilingual Children. Dordrecht, Foris.

CORDIER, F. (1994), Représentation cognitive et langage : une conquête progressive, Paris, Armand Colin.

DUBOSQ-SÉNÉMAUD, M. (1997), L'enfant et le récit : étude sur la temporalité dans des récits d'enfants en vue d'une recherche sur les récits d'enfants dysphasiques, Mémoire de maitrise, Université Paris X, Sciences du Langage.

KERN, S. (1997), Comment les enfants jonglent avec les contraintes communicationnelles, discursives et linguistiques dans la production d'une narration, Thèse de doctorat, Université Lumière-Lyon 2, Sciences du Langages.

KLEIN, W. (19872), Zweitspracherwerb. Eine Einführung, Frankfurt am Main, Athenäum, (Trad. franç. de C. Noyau (1989), L'acquisition de langue étrangère, Paris, Armand Colin).

KLEIN, W. (1994), Time in Language, London, New York, Routledge.

LUTHER, M., « Missive sur la traduction » (Trad. de l'allemand par H. Guicharrousse), in CEuvres, Paris, Gallimard, 'La Pléiade' (à paraître).

MAYER, M. (1980/1969), Frog, Where Are You? New York, Dial Book for Young Readers.

SABEAU-JOUANNET, E. (1977), « L'expression de l'organisation spatiale et temporelle, son évolution chez des enfants de 2 à 5 ans ", chapitre IX de François, F., Fançois, D., Sabeau-Jouannet, E. \& Sourdot, M. La syntaxe de l'enfant avant cinq ans, Paris, Larousse.

RONJAT, J. (1913), Le développement du langage observé chez un enfant bilingue, Paris, Champion. 
WEIST, R. M. (1989), “Time Concepts in Language and Thought: Filling the Piagetian Void from Two to Five Years”, in Levin, I. \& Zakay, D. (éds.) Time and Human Cognition. A Life-Span Perspective, North-Holland, Elsevier Science Publishers B.V.

\section{NOTES}

1. Depuis le conseil donné par le linguiste Grammont à Jules Ronjat pour l'éducation bilingue de son fils, ce principe est connu sous le nom de « une langue - une personne ». Cf. Ronjat (1913:3).

2. L'équilibre entre les deux langues s'est établi vers 3 ans. A partir de cet âge il est même difficile de parler de la dominance d'une langue sur l'autre.

3. $2 ; 8$ se lit 2 ans 8 mois.

4. Jusque là, l'enfant avait recours à un certain nombre d'éléments français dans ses énoncés pour satisfaire ses besoins communicatifs avec sa mère, dont il savait qu'elle connaissait le français [ex. : Tu schreib quoi auf ? $(2 ; 6)$ ].

5. Dans la mesure où ces structures sont apparues en même temps en allemand (vers $2 ; 8$ ), nous commencerons par inventorier celles-ci par commodité et nous ferons ensuite un retour en arrière pour analyser quelques précurseurs et la subordination causale en [paske] qui est apparue plus précocément en français.

6. Entre parenthèses figurent les traductions en français des exemples allemands et entre crochets les interprétations et commentaires.

7. Après et surtout son équivalent allemand danach ont rempli dans la grammaire de l'enfant plusieurs fonctions transitoires que nous analyserons ci-dessous.

8. Dans la subordonnée allemande le verbe fléchi se trouve en position finale, et dans la principale les positions du sujet et de la composante fléchie sont inversées si la subordonnée précède la principale. $\mathrm{Cf}$. aussi l'article de Vera Führer-Nicod dans ce numéro pour un aperçu des règles grammaticales en allemand dans le domaine de la subordination temporelle.

9. Cf. Klein (1987) pour les règles et le développement du placement des formes verbales fléchies (FIN) et non fléchies (INF) en allemand.

10. - assertive avec complément : Comp V S (ex. : Morgen komme ich.)

- question ouverte : Interrogatif V S (ex. : Wann kommst du ?)

- question fermée : V S (ex. : Kommst du ?)

11. Cf. Klein (1994) pour une discussion des notions de temps absolu (le relatum est le moment de parole) et de temps relatif (le relatum est un moment-repère quelconque).

12. Cf. Sabeau-Jouannet (1977:196) sur l'opposition antériorité/postériorité et le problème du temps social dans le domaine de l'acquisition de l'expression de la temporalité.

13. Cf. Cordier (1994) pour les définitions de ces deux catégories et le débat sur l'ordre de développement de ces deux catégories l'une par rapport à l'autre.

14. Comme pour les autres subordonnées en allemand, la structure en weil requiert la position finale de la forme verbale fléchie.

15. Cet usage semble être admis en allemand familier. On en trouve d'ailleurs trace dans des textes littéraires, notamment d'auteurs tels que Bertolt Brecht par exemple, qui font comme le préconisait Martin Luther pour sa traduction de la Bible : « man muß [dem Volk] auff das maul sehen (il faut [...] regarder ceux-ci [i.e. le peuple] à la gueule ; trad. de H. Guicharrousse) ».

16. L'enfant qui commence à manier ces relations entre dans ce que Weist (1989) appelle "Reference Time System". cf. l'article de S. Romeo ici même.

17. Cf. Dubosq-Sénémaud (1997:37).

18. Cf. Kern (1997:291). 
19. A la suite de Berman \& Slobin (1994) cette histoire de Mayer (1969) en 24 images sans paroles sert de support à de nombreuses études translinguistiques sur la compétence narrative chez enfants et adultes.

20. Cf. Bamberg (1994), qui a fait le même constat pour les enfants germanophones de 3 ans.

\section{RÉSUMÉS}

Cet article présente une étude de cas portant sur l'acquisition de la subordination temporelle par un enfant bilingue français-allemand et couvrant la période entre 2 ans 2 mois et 3 ans 4 mois. En partant des structures linguistiques observables dans les données, l'étude détermine les étapes d'émergence des différentes structures dans les deux langues ainsi que leurs précurseurs et les relations temporelles exprimées (concomitance, antériorité, postériorité). L'objectif de l'analyse était également de préciser dans quels contextes discursifs la subordination temporelle fait ses premières apparitions et se trouve le plus souvent employée (conversation, récit de vie, récit à partir d'images). Les résultats montrent que la subordination temporelle est d'abord employée dans la conversation courante, puis dans les récits d'expériences vécues par l'enfant, pour n'apparaître que plus tardivement dans les récits produits à partir d'images. Son acquisition et son emploi suivent un développement quasi-parallèle dans les deux langues.

This case study investigates the acquisition of temporal subordinate clauses by a French-German bilingual child between the age of 2 years 2 months and 3 years 4 months. Taking the linguistic structures observed in the data as a basis of analysis, the study determines the developmental stages of the emergence of different structures in the two languages concerned as well as their precursors and the temporal relations expressed. Particular attention is paid to the discourse contexts in which temporal subordinate clauses first appear and are most frequently used (conversation, narratives, picture-story telling). The results show that temporal subordinate clauses first appear in free conversation, then in narratives about the subject's personal experience and only later in picture-story telling. The acquisition and the use of temporal subordinate clauses follow an almost parallel development in both languages.

\section{AUTEUR}

\section{RICARDA SCHNEIDER}

Université Paris X-Nanterre EA 372 Modèles Linguistiques et Dynamiques des Langues et Groupement de Recherche 113 du CNRS, Ricarda.Schneider@u-paris10.fr 